\title{
La monnaie dans les discours du CNPF entre 1947 et 1969 : un révélateur des relations entre le patronat et les gouvernements
}

\section{Béatrice Touchelay}

\section{OpenEdition}

\section{Journals}

Édition électronique

URL : http://journals.openedition.org/ress/239

DOI : $10.4000 /$ ress.239

ISSN : 1663-4446

Éditeur

Librairie Droz

\section{Édition imprimée}

Date de publication : 1 juillet 2007

Pagination : 233-250

ISBN : 978-2-600-01155-6

ISSN : 0048-8046

Référence électronique

Béatrice Touchelay, "La monnaie dans les discours du CNPF entre 1947 et 1969 : un révélateur des relations entre le patronat et les gouvernements », Revue européenne des sciences sociales [En ligne], XLV-137 | 2007, mis en ligne le 01 juillet 2010, consulté le 20 avril 2019. URL : http:// journals.openedition.org/ress/239; DOI : 10.4000/ress.239 


\section{LA MONNAIE DANS LES DISCOURS DU CNPF ENTRE 1947 ET 1969: Un révélateur des relations entre le patronat et les gouvernements}

L'élimination des protections douanières et la modernisation économique constituent des faits majeurs pour la France des années 1947 à 1969. Deux discours s'affrontent pour expliquer ces évolutions. Le premier les attribue au volontarisme de l'Etat à partir des réformes structurelles de la Libération. Le second considère que ces évolutions s'imposent malgré l'intervention des pouvoirs publics.

Le Conseil National du Patronat Français fondé en 1946 définit ses positions de façon pragmatique ${ }^{1}$. Il tient compte des intérêts contradictoires de ses affiliés pour se prononcer sur le désarmement douanier puis sur la construction européenne. Les dirigeants des principales fédérations (sidérurgie et textile) sont peu favorables à l'ouverture. Ils souhaitent maintenir les protections traditionnelles alors que la majorité des entreprises appartient au secteur abrité et veut limiter les charges et les réglementations. Les éléments modernisateurs du patronat sont dépassés par une majorité qui reste frileuse ${ }^{2}$.

Le CNPF dénonce un Etat omnipotent qui sacrifie la valeur du franc aux facilités de l'inflation et des déficits budgétaires et qui renonce à défendre l'entreprise française dans la concurrence étrangère. Son discours s'adapte aux évolutions des politiques économiques. Trois périodes peuvent être distinguées.

Dans un premier temps, la critique des interventions de l'Etat est systématique. Le patronat est sur la défensive. La crise de la France à l'UEP et la formation du gouvernement Pinay au début de 1952 infléchissent le discours. Le CNPF cherche désormais à développer le dialogue avec les pouvoirs publics. Il demande d'aider les entreprises à résister au désarmement douanier. L'interruption du processus de libéralisation des échanges puis le maintien de protections soulagent les dirigeants patronaux. Ces mesures sont pourtant en parfaite contradiction avec la condamnation de l'interventionnisme.

Le discours patronal retrouve une certaine cohérence avec la formation de la $\mathrm{V}^{\mathrm{e}}$ République. Le CNPF est satisfait par la réforme monétaire et le retour des équilibres extérieurs mais il continue à réclamer des aides de l'Etat pour favoriser l'intégration des entreprises dans la compétition internationale. La réforme des statuts du CNPF en 1969 clôt la période des fondations. Le patronat et les gouvernants sont désormais en relations constantes. Le consensus est consolidé par la solidité du franc. Il n'est pas ébranlé par la dévaluation qui achève la période.

\footnotetext{
Ehrmann (1959); Weber (1996).

2 Touchelay (avril-juin 2000).
} 
Le Bulletin du CNPF jusqu'à 1960 puis Patronat français commente ces évolutions et présente les positions des instances dirigeantes du CNPF. Les éditoriaux des présidents Georges Villiers jusqu'à 1965 et Pierre Huvelin ensuite donnent le ton.

\section{LE FRANC, \\ SYMBOLE DE L'INEFFICACITÉ DE L'ETAT \\ POUR UN PATRONAT \\ SUR LA DÉFENSIVE (1947-1951)}

Au cours de l'assemblée générale statutaire du 7 décembre 1946, le CNPF charge un bureau, un comité directeur et des commissions spécialisées de définir et de diffuser ses positions ${ }^{3}$. Georges Villiers fait de l'augmentation des prix intérieurs et du déséquilibre budgétaire des symboles de l'inefficacité des interventions des pouvoirs publics. Il définit sans le savoir les positions patronales des vingt années suivantes.

Les articles du Bulletin du CNPF sur les relations économiques extérieures diffusent des thèses libérales ${ }^{4}$. Ils prônent la libéralisation des échanges et des changes. Le CNPF demande d'harmoniser les conditions de production et de résoudre les problèmes monétaires avant d'éliminer les protections douanières entre pays. Il condamne les négociations entamées par les gouvernements français et italiens en février 1948 pour former une union douanière. Les pressions du CNPF contribuent au report du projet ${ }^{5}$. Outre ces pressions, le CNPF cherche à limiter les contraintes administratives imposées aux entreprises. Il organise des journées d'étude de l'exportation afin que les producteurs nouent des contacts avec les représentants des administrations puis il charge une sous commission de l'examen des formalités administratives.

Les responsables du CNPF invitent les chefs d'entreprises à développer l'exportation et à se préparer à vivre sans l'aide américaine. Georges Villiers multiplie les initiatives pour faire connaître les productions françaises aux Etats-Unis. Il participe aux manifestations organisées par les industriels américains puis il implique le CNPF dans la création du comité franc dollar. Un tiers des éditoriaux du Bulletin du CNPF de l'année 1948 rappelle l'obligation d'exporter ${ }^{6}$. L'appel est soutenu par les présidents des principales commissions patronales. Ils insistent d'autant plus sur les chiffres « angoissants » du commerce extérieur que le processus de diminution des droits de douane des principaux pays industriels est

3 Compte rendu de l'assemblée générale statutaire du 7 décembre 1946, Bulletin du CNPF, $\mathrm{n}^{\circ} 3$, février 1947. Les commissions les plus importantes pour notre sujet : commission de l'économie générale présidée par Pierre Ricard, commission des accords internationaux présidée par Jean-Jacques Guerlain et commission des prix dirigée par Henri Fayol.

4 Ch. Rist, « Liberté des changes », Bulletin du CNPF, $\mathrm{n}^{\circ}$ 10, novembre 1947. Extrait de La Vie française du 31 octobre 1947.

5 R. Arnaud, «Le projet d'union douanière franco-italienne », Bulletin du CNPF, $\mathrm{n}^{\circ} 25,1^{\mathrm{er}}$ janvier 1949 ; Ricard, Pierre, «L'union douanière franco-italienne », Id., nº 27, $1^{\text {er }}$ février 1949.

6 G. Villiers, « Comment développer nos exportations ? », Bulletin du CNPF, $\mathrm{n}^{\circ} 17$, juillet 1948 ; «Développer la production et les exportations », Id., $\mathrm{n}^{\circ} 18,1^{\text {er }}$ septembre 1948 ; «Comment doubler nos exportations? », Id., $\mathrm{n}^{\circ} 24,15$ décembre 1948. 
enclenché ${ }^{7}$. Les appels à l'exportation s'adressent essentiellement aux chefs d'entreprises jusqu'à la fin de l'année 1948. A partir de cette date, les dirigeants du CNPF interpellent les pouvoirs publics. Ils leur réclament de soutenir les exportateurs sans avoir à rétablir les droits de douane ${ }^{8}$.

L'assemblée générale de décembre 1948 marque le début de l'offensive contre la politique gouvernementale. Jean-Jacques Guerlain considère que les charges, les lourdeurs administratives et les restrictions en matière de devises freinent « l'action méritoire des exportateurs ${ }^{9}$. Les éditoriaux de Georges Villiers développent ces critiques. Ils condamnent le dérapage des tarifs publics et du budget. Le manque de fermeté des responsables politiques dans les négociations salariales est accusé d'alourdir les prix français et de handicaper les entreprises confrontées aux contraintes extérieures ${ }^{10}$. Le comité directeur du CNPF dénonce également l'inefficacité des subventions et des soutiens à la productivité ${ }^{11}$. Le Bulletin du $C N P F$ diffuse ces critiques. Elles sont relayées auprès des Chambres et dans les administrations concernées par des commissions ou des comités d'études dans lesquels interviennent les professionnels.

L'intervention de Georges Villiers à l'assemblée générale de juillet 1949 révèle la faiblesse relative de l'influence du CNPF sur la décision politique ${ }^{12}$. Il attribue aux pressions patronales la suppression de nombreuses taxations mais il dénonce l'existence de produits nationalisés qui bloque les mécanismes de la concurrence. Quelques semaines plus tard, Georges Villiers conteste le bienfondé du remaniement monétaire de septembre $1949^{13}$. La date du remaniement est critiquée parce qu'elle correspond à la fin d'une longue dépression monétaire. Georges Villiers précise toutefois que ses effets dépendront « des marchés internationaux ». Comme il ne s'agit pas d'une dévaluation générale, ses effets seront vraisemblablement limités. Le CNPF intervient directement auprès des Autorités compétentes pour qu'elles soutiennent les importateurs de produits de la zone dollar après la dévaluation ${ }^{14}$. Il entame parallèlement une campagne d'opinion afin que les pouvoirs publics aident les industriels français à surmonter la première étape de la suppression des contingentements prévue par l'OECE le $1^{\text {er }}$ octobre

7 J.-J. Guerlain, «Intervention à l'assemblée générale du 19 décembre 1947 », Bulletin du CNPF, $\mathrm{n}^{\circ} 11$, janvier 1948 .

${ }^{8}$ Compte rendu de la réunion du comité directeur du 19 novembre, Bulletin du CNPF, $\mathrm{n}^{\circ} 23,1^{\mathrm{er}}$ décembre 1948 .

9 J.-J. Guerlain, « Travaux de la commission des échanges internationaux », Bulletin du CNPF, $\mathrm{n}^{\circ} 25,1^{\mathrm{er}}$ janvier 1949.

${ }^{10} \mathrm{G}$. Villiers, «A propos des récentes mesures gouvernementales », Bulletin du CNPF, $\mathrm{n}^{\circ} 26$, 15 janvier 1949 ; « Du dirigisme à la liberté », Id., $\mathrm{n}^{\circ} 27,1^{\text {er }}$ février 1949 ; « Productivité », Id., n 30 , 15 mars 1949 ; «L'abondance présente-t-elle des risques ? », Id., n 32, 15 avril 1949 ; « La libération des salaires », Id., n 33, $1^{\text {er }}$ mai 1949 ; « Les devoirs de l’Etat », Id., n 34, 15 mai 1949.

${ }^{11}$ Compte rendu de la réunion du comité directeur du 15 février, Bulletin du CNPF, $\mathrm{n}^{\circ} 29$, $1^{\mathrm{er}}$ mars 1949.

12 G. Villiers, «Intervention du $1^{\text {er }}$ juillet $1949 »$, Bulletin du CNPF, $\mathrm{n}^{\circ} 36$, juillet-août 1949.

13 G. Villiers, « Les conséquences de l'alignement monétaire », Bulletin du CNPF, $\mathrm{n}^{\circ}$ 37, septembre 1949 .

${ }^{14}$ Compte rendu de travaux, Bulletin du CNPF, $\mathrm{n}^{\circ} 38$, octobre 1949. 
1949. Le CNPF réclame une réforme générale, des économies budgétaires, l'amélioration de la gestion du secteur nationalisé et l'allégement des frais généraux de la nation. Ces revendications sont transmises au ministre des Finances Edgar Faure et à la presse. Dans le Bulletin du CNPF, Georges Villiers rappelle que le patronat ne soutient la libéralisation des échanges et la formation d'une union européenne que si elles s'accompagnent du rétablissement de la libre convertibilité des monnaies et de l'harmonisation des politiques des pays concernés. Il condamne l'alourdissement du «fardeau fiscal » et la persistance du « dirigisme ${ }^{15}$. Deux éditoriaux, une motion du comité directeur réuni le 15 novembre 1949 puis une nouvelle lettre ouverte au ministre des Finances le $1^{\text {er }}$ décembre soulignent clairement que les conditions du soutien patronal à l'ouverture ne sont pas réunies ${ }^{16}$. Le CNPF dénonce en particulier les pratiques non concurrentielles entre pays européens. Il vise à la fois les importations de choc destinées à forcer la baisse des prix intérieurs et les pratiques discriminatoires allemandes.

Devant l'assemblée générale de janvier 1950, Georges Villiers évoque les pressions quotidiennes exercées par le CNPF auprès des pouvoirs publics français pour que les producteurs disposent des mêmes conditions de concurrence que leurs partenaires européens ${ }^{17}$. En demandant d'intégrer les professionnels aux négociations commerciales et de faire de la libéralisation des échanges l'axe central de toute politique, Georges Villiers définit la stratégie du CNPF pour les deux années à venir. Chaque décision gouvernementale est évaluée à l'aune des échéances d'ouverture des frontières. Ainsi, par exemple, si le CNPF est très favorable au rétablissement de la libre négociation des salaires décidé par la loi de février 1950, il réclame aux pouvoirs publics de tout mettre en œuvre pour limiter les pressions salariales et pour ne pas alourdir les prix de revient ${ }^{18}$. En mars 1950, le comité directeur dresse un bilan négatif des premières étapes de la réduction des contingentements ${ }^{19}$. Il critique «l'arbitraire » de la liste des produits libérés ainsi que le maintien des contrôles administratifs. Il dénonce avec force la poussée des pressions salariales. Il condamne enfin les augmentations des taxes et des charges qui sont prévues dans le budget de $1951^{20}$. Le groupement patronal demande d'accompagner la baisse des contingentements par le rétablissement de certains droits de douane. Il renforce ses groupes d'études en formant une commission du libre-échange et en réorganisant ses commissions spécialisées dans les questions 1949.

15 G. Villiers, « La libération des échanges à l'ordre du jour », Bulletin du $C N P F, \mathrm{n}^{\circ} 39$, novembre

16 G. Villiers, «Economie et fiscalité », Bulletin du CNPF, $\mathrm{n}^{\circ} 40,1^{\text {er }}$ décembre 1949 ; « A propos des impôts », Id. , n 41, 15 décembre 1949.

17 G. Villiers, « Exposé sur la situation économique générale devant l'assemblée générale », Bulletin du CNPF, $\mathrm{n}^{\circ}$ 44, 5 février 1950.

${ }^{18}$ G. Villiers, « Le patronat et les investissements », Bulletin du CNPF, $\mathrm{n}^{\circ} 42,5$ janvier 1950 ; «Le niveau des salaires », Id., $\mathrm{n}^{\circ} 43,20$ janvier 1950 ; « Profession ou politique », Id., $\mathrm{n}^{\circ} 45,20$ février 1950 ; «Salaires et équilibre économique », Id., n 46, 5 mars 1950 ; « Les salaires, les ressources et la production », $I d$., $\mathrm{n}^{\circ} 48,5$ avril 1950.

19 Compte rendu des travaux du CNPF, Bulletin du CNPF, $\mathrm{n}^{\circ} 46,5$ mars 1950.

${ }^{20}$ Travaux du comité directeur, Bulletin du CNPF, n 47, 20 mars 1950 (texte communiqué à la presse) ; G. Villiers, « Réponses à un homme de bonne foi », Id., n 50, 5 mai 1950 ; « Avant qu'il ne soit trop tard », Id., $\mathrm{n}^{\circ}$ 52, 20 juin 1950. 
extérieures $^{21}$. Certaines revendications patronales sont satisfaites. Le remboursement partiel des charges aux exportateurs ou encore la définition des mécanismes de l'UEP constituent de solides avancées mais elles ne suffisent pas à infléchir la position des dirigeants patronaux à l'égard des gouvernants. Ils confirment leur défiance en rejetant le projet Schuman et ils prolongent leur offensive contre la pression fiscale jusqu'au début de $1951^{22}$.

Devant l'assemblée générale de janvier 1951, Georges Villiers précise les critiques du patronat contre la proposition Schuman ${ }^{23}$. En juillet 1951, Pierre Ricard confirme son opposition à ce projet « qui expose l'industrie lourde française à des dangers certains au bénéfice d'une construction purement idéologique ${ }^{24}$. La plupart des éditoriaux de 1951 critiquent les décisions économiques des pouvoirs publics. En février, par exemple, Georges Villiers reproche aux gouvernants de ne pas tenir leurs promesses et de continuer à alourdir les charges des exportateurs ${ }^{25}$. Il dénonce les fortes rigidités salariales maintenues par la présence de la fonction publique et par le mécanisme de l'échelle mobile malgré le retour à la libre négociation des salaires ${ }^{26}$. Georges Villiers considère que les charges fiscales et sociales affaiblissent les producteurs français et leur imposent des « conditions de production » défavorables par rapport à celles de leurs partenaires étrangers ${ }^{27}$. Toute augmentation des charges est jugée menacer les chances de la France de participer à la construction européenne ${ }^{28}$. Pour avoir imposé une fiscalité «injuste et anti-économique» au lieu de défendre la monnaie « avec le courage nécessaire », les gouvernements sont acculés à employer des « expédients provisoires $»^{29}$. Le CNPF souligne qu'il refuse de se satisfaire de l'amélioration des aides ou des remboursements de certaines charges aux exportateurs. Le maintien de quelques protections douanières ne suffira pas davantage à améliorer la situa-

${ }^{21}$ Compte rendu de l'assemblée générale du $1^{\mathrm{er}}$ juillet 1950, Bulletin du $C N P F, \mathrm{n}^{\circ} 54,20$ juillet5 août 1950. La commission du commerce extérieur devient celle de l'expansion commerciale. La commission des relations économiques internationales et celle des ententes internationales sont créées.

22 Ibid. P. Ricard précise que le plan Schuman risque de renforcer « l'intervention de l'Etat en matière économique »; G. Villiers, « Le problème des matières premières », $I d$., $\mathrm{n}^{\circ} 58,20$ novembre 1950 ; «Impôts nouveaux », Id., n 59,5 décembre 1950 ; «Pour un climat d'expansion économique », Id., $\mathrm{n}^{\circ} 61,20$ janvier 1951.

${ }^{23}$ G. Villiers, « Exposé sur les questions économiques internationales », Bulletin du $C N P F, \mathrm{n}^{\circ} 62$, 19 janvier 1951. Georges Villiers dénonce les «pouvoirs discrétionnaires confiés pour cinquante ans à une Haute autorité de six techniciens...».

${ }^{24}$ Compte rendu des travaux de l'assemblée générale de juillet 1951, Bulletin du CNPF, ${ }^{\circ} 69$, août 1951.

${ }^{25}$ G. Villiers, « Nécessité de définir une politique économique », Bulletin du $C N P F, \mathrm{n}^{\circ} 63$, 20 février 1951.

${ }^{26}$ G. Villiers, « Salaires minimum garantis et convention collective », Bulletin $d u C N P F, \mathrm{n}^{\circ} 65$, 20 mars-5 avril 1951.

27 G. Villiers, « A propos des pénalités fiscales », Bulletin du CNPF, n 67, 20 mai-5 juin 1951.

${ }^{28}$ G. Villiers, «Conséquences d'une fiscalité excessive », Bulletin du CNPF, $\mathrm{n}^{\circ} 70,5$ septembre 1951 ; « Salaires, prix, inflation », Id., n 71, 20 septembre-5 octobre 1951.

${ }^{29}$ G. Villiers, « Perspectives budgétaires », Bulletin du CNPF, $\mathrm{n}^{\circ} 73,5$ novembre 1951. 
tion des exportateurs français handicapés par le prix des matières premières et par «les augmentations des prix intérieurs $»^{30}$.

La rapide dégradation de la position française à l'UEP confirme les inquiétudes du patronat. La formation surprise du gouvernement d'Antoine Pinay change la nature des relations entre le CNPF et les autorités politiques.

\section{LE FRANC, D'UN ASSAINISSEMENT À LA RÉFORME MONÉTAIRE : LE CHANGEMENT D'ATTITUDE DU PATRONAT (1952-1958)}

A la veille de la formation du gouvernement d'Antoine Pinay, le discours patronal est des plus sombres. Pierre Ricard reproche aux pouvoirs publics d'avoir provoqué la crise française à l'UEP en diminuant les droits de douane sur les produits manufacturés sans consulter les organisations industrielles ${ }^{31}$. Fin janvier 1952, la situation extérieure de la France est alarmante. Le 3 février 1952, le gouvernement suspend les importations. L'éditorial du Bulletin du CNPF du 5 février dénonce le poids des charges et des salaires qui empêche les « entreprises françaises » de résister à l'ouverture des frontières ${ }^{32}$. Comme le souligne le rapport du sénateur Armengaud sur le pool charbon acier, la supériorité des prix de revient français relève de décisions politiques. Inspiré par ce rapport, le comité directeur du CNPF s'oppose à la création du pool tant que les pouvoirs publics ne prendront aucune mesure pour atténuer la supériorité des salaires français.

Le ton du discours patronal change radicalement à partir du 6 mars 1952. Antoine Pinay forme son gouvernement. Il s'engage à défendre le franc et à rétablir les grands équilibres. Il est encouragé par plusieurs éditoriaux de Georges Villiers. Le premier propose des remèdes à la crise française à l'UEP ${ }^{33}$. Il rejette la facilité en écartant la dévaluation. Il préconise d'intervenir sur les prix de revient afin de « remettre la production française en état de concurrencer la production étrangère ». Pour être efficace, le gouvernement doit alléger la fiscalité et les charges sociales et réduire le coût du crédit et de l'énergie. L'éditorial du mois d'avril demande de compléter la « courageuse » politique des prix intérieurs par une augmentation des aides à l'exportation ${ }^{34}$. Georges Villiers invite ensuite le gouvernement à approfondir le changement par des « réformes profondes » et il appelle les producteurs à le soutenir ${ }^{35}$. La confiance du CNPF dans le gouverne-

${ }^{30}$ G. Villiers, « Les entreprises françaises en péril », Bulletin du CNPF, n 75, 20 décembre 1951.

${ }^{31} \mathrm{P}$. Ricard, «Exposé sur les questions économiques internationales devant l'assemblée générale du 12 janvier $1952 »$, Bulletin du CNPF, n 78, 20 février 1952.

${ }^{32}$ G. Villiers, « Une situation catastrophique », Bulletin du CNPF, n 77, 5 février 1952 (texte communiqué à la presse).

${ }^{33}$ G. Villiers, « La défense du franc », Bulletin du CNPF, n 79, 5-20 mars 1952.

${ }^{34}$ G. Villiers, « La nécessité d'exporter », Bulletin du CNPF, n 80, 5 avril 1952 (texte de l'allocution radiodiffusée du $1^{\text {er }}$ avril 1952).

35 G. Villiers, «La baisse des prix, devoir des entreprises et des professions », Bulletin du CNPF, n 81, 5 mai 1952; «L'emprunt doit réussir », Id., n 82, 5 juin 1952. 
ment Pinay est confirmée lors de l'assemblée générale de juin $1952^{36}$. Elle favorise le changement d'attitude du patronat à 1 'égard du plan Schuman.

Tout en critiquant le principe du blocage des prix décidé fin août, le CNPF continue à accorder sa confiance aux autorités politiques ${ }^{37}$. Il maintient ses pressions pour obtenir une augmentation des aides à l'exportation et une baisse des prix de revient français. Le 21 octobre 1952, une délégation présente les solutions patronales susceptibles de restaurer l'équilibre commercial à la présidence du Conseil $^{38}$. Constatant que les salaires français sont les «seuls au monde à subir une majoration de $5 \%$ sous forme d'impôts », elle suggère de limiter les dépenses de la Sécurité sociale en réformant sa gestion et de revenir sur certaines charges salariales comme « la majoration des heures supplémentaires ».

A partir de décembre 1952, le CNPF demande aux successeurs du gouvernement Pinay de prolonger une politique qui a redressé le solde commercial français en rendant aux entreprises « leurs possibilités normales d'exporter ${ }^{39}$. Le CNPF réclame de continuer à limiter l'inflation, à élargir les conditions du crédit et à augmenter le remboursement des charges des exportateurs afin de rétablir durablement les finances extérieures ${ }^{40}$. Georges Villiers incite également les organisations patronales à renforcer leurs liens pour réagir à l'instabilité gouvernementale $^{41}$. Au cours de l'assemblée générale de juillet 1953, il invite le CNPF à ne plus être «seulement un organisme de défense » mais à se prononcer sur les grands problèmes nationaux ${ }^{42}$. La majorité des affiliés est d'autant moins favorable à un changement de stratégie que les mouvements sociaux de l'été 1953 puis la hausse générale des salaires accordée en septembre accentuent les inquiétudes ${ }^{43}$.

A partir de 1954, le CNPF dénonce la supériorité des charges des industriels français sur celles des concurrents européens ${ }^{44}$. Il condamne les réactions « artificielles » des gouvernements qui renforcent les protections douanières alors que les autres pays « suppriment leurs contingentements ». Parallèlement, Georges Villiers souhaite élargir les ambitions du CNPF. Il lui confie trois objectifs en

${ }^{36}$ Compte rendu de l'assemblée générale du 27 juin 1952, Bulletin du CNPF, $\mathrm{n}^{\circ}$ 84, 20 juillet5 août 1952. Exposé de G. Villiers : «L'espoir renaît »; P. Ricard, Id. : la sidérurgie française « apportera sa collaboration confiante » à la Haute Autorité.

${ }^{37}$ G. Villiers, «Continuité de l'effort », Bulletin du CNPF, n 85, 5-20 septembre 1952 ; « Réforme fiscale », $I d$., n $86,5-20$ octobre 1952 (texte transmis à la presse).

${ }^{38}$ H. Fayol, « Les causes des disparités des prix français et étrangers », supplément au Bulletin du $C N P F, \mathrm{n}^{\circ} 88,20$ novembre 1952.

${ }^{39}$ G. Villiers, Sans titre, Bulletin du CNPF, n 91, 5 janvier 1953 ; Numéro spécial assemblée générale, $I d ., \mathrm{n}^{\circ}$ 92, 20 janvier 1953.

${ }^{40}$ Compte rendu de la réunion du comité directeur, Bulletin du CNPF, n ${ }^{\circ}$ 94, 20 février-5 mars 1953 ; G. Villiers, « Plein emploi et assainissement de l'économie sont des objectifs inséparables », Id., $\mathrm{n}^{\circ} 95,20$ mars 1953.

${ }^{41}$ G. Villiers, «Les chefs d'entreprises devant les problèmes actuels », Bulletin du CNPF, $\mathrm{n}^{\circ} 101$, 20 juin 1953.

42 G. Villiers, «Intervention », Bulletin du CNPF, $\mathrm{n}^{\circ}$ 103, 20 juillet 1953.

${ }^{43}$ G. Villiers, «Les raisons de la position patronale », Bulletin du CNPF, $\mathrm{n}^{\circ} 105,5-20$ septembre 1953 ; Compte rendu des réunions du comité directeur, $I d ., \mathrm{n}^{\circ} 108,5$ novembre 1953 et $\mathrm{n}^{\circ} 110,5$ décembre 1953.

${ }^{44} \mathrm{G}$. Villiers, « Niveau de vie et production nationale », Bulletin $d u C N P F, \mathrm{n}^{\circ} 112,5$ janvier 1954. 
janvier 1954 : « augmenter le niveau de vie des français, augmenter la productivité nationale, rendre les prix compétitifs $»^{45}$. L'attention de la majorité des producteurs est limitée au très court terme puisque la première étape de baisse des protections douanières est prévue le $1^{\mathrm{er}}$ mars 1954. Le CNPF juge cette échéance insurmontable malgré la promesse du ministre des Finances Edgar Faure de baisser les charges. La revalorisation des plus bas salaires décidée le 5 février 1954 déclenche une fronde patronale ${ }^{46}$. Le comité directeur condamne cette décision « autoritaire » et incompatible avec la libéralisation des échanges. Le blocage des prix décidé le 17 février 1954 ne convient pas davantage. En mars 1954, Georges Villiers pense qu'il faudra interrompre le processus d'ouverture des frontières comme en 1952 si aucune réforme n'est entreprise ${ }^{47}$. Malgré ces mises en garde, la situation des finances extérieures continue de se dégrader.

En mai 1954, Georges Villiers approuve l'engagement français de diminuer en deux étapes (le 15 avril puis le $1^{\text {er }}$ novembre) les restrictions aux importations issues de l'OECE ${ }^{48}$. Il rappelle toutefois que ces échéances seront insupportables si le poids des charges, la dépendance à l'égard des importations des matières premières et le contrôle des prix propres à la France ne sont pas éliminés ${ }^{49}$. La prorogation du mécanisme de l'UEP jusqu'au 30 juin 1955 est le seul sujet de satisfaction des exposés sur la situation extérieure présentés à l'assemblée générale de juillet $1954^{50}$. Aucun des trois objectifs impartis au CNPF en 1953 n'a progressé : la production stagne, l'amélioration du niveau de vie comme celle de la compétitivité provient des « artifices provisoires » du blocage des prix et des compensations. Georges Villiers accuse les gouvernements d'acculer le CNPF à réclamer des aides pour surmonter les échéances de la libéralisation des échanges alors qu'il est profondément convaincu de leur caractère artificiel.

La première étape de la baisse des contingents fixée au 15 avril est franchie sans drame. Le CNPF reporte ses inquiétudes sur la seconde étape du $1^{\text {er }}$ novembre et pour laquelle la France bénéficie d'un taux privilégié. Georges Villiers lui consacre trois éditoriaux entre août et octobre $1954^{51}$. Il réclame à nouveau d'éliminer le blocage des prix, de limiter les hausses de salaires, d'alléger les charges et d'améliorer l'approvisionnement en matières premières.

Paradoxalement, l'éditorial de novembre ne traite pas de l'échéance du $1^{\mathrm{er}}$ novembre mais des problèmes de l'Union française ${ }^{52}$. Les catastrophes annoncées

45 Compte rendu de l'assemblée générale du 19 janvier, Bulletin du CNPF, n 114, 5 février 1954.

46 Travaux du comité directeur, Bulletin du CNPF, n 116, 5 mars 1954.

47 G. Villiers, «Conditions et perspectives d'une politique de libéralisation des échanges », Bulletin du CNPF, $\mathrm{n}^{\circ}$ 117, 20 mars 1954 : «En raison de l'urgence de la question, le texte est communiqué à la presse ».

${ }^{48}$ G. Villiers, «L'économie française devant des échéances inéluctables », Bulletin du CNPF, $\mathrm{n}^{\circ} 119$, mai 1954.

${ }^{49}$ G. Villiers, Sans titre, Bulletin du CNPF, $\mathrm{n}^{\circ} 120$, juin 1954 ; « La situation des échanges extérieurs reste préoccupante $», I d$., $\mathrm{n}^{\circ} 121$, juillet 1954.

${ }^{50}$ Compte rendu de l'assemblée générale du 6 juillet, Bulletin du $C N P F, \mathrm{n}^{\circ} 122$, août 1954.

${ }^{51}$ G. Villiers, «L'échéance du $1^{\mathrm{er}}$ novembre », Bulletin du CNPF, $\mathrm{n}^{\circ} 123$, août 1954 (communiqué à la presse) ; «La concurrence internationale, impératif d'une politique économique cohérente », Id., $\mathrm{n}^{\circ} 124$, septembre 1954 ; « Les hausses de salaires », Id., $\mathrm{n}^{\circ} 125$, octobre 1954.

52 G. Villiers, «Les problèmes de l'Union française », Bulletin du CNPF, n 126 , novembre 1954. 
par le CNPF pour surmonter les premières étapes de l'ouverture des frontières ne se produisent pas. Il conserve pourtant les mêmes mots d'ordre ${ }^{53}$.

Au début de 1955, Georges Villiers appelle toutes les « élites françaises » à contribuer à résoudre les difficultés : il souhaite que les pouvoirs publics réforment les institutions, que les chefs d'entreprises accentuent leurs efforts pour insérer la France dans « la compétition internationale » et que le CNPF ne se limite plus à la défense de l'entreprise privée mais diffuse davantage d'informations sur les problèmes de l'industrie ${ }^{54}$. Une fois de plus, l'appel de Georges Villiers est sans effet immédiat. Il ne le reprend pas à l'assemblée générale de janvier 1955 mais consacre son discours à l'échéance de baisse des contingentements du $1^{\mathrm{er}}$ avril $1955^{55}$. Le décret contre la hausse générale des prix ne rassure pas davantage que l'augmentation du remboursement des charges des exportateurs. La forte croissance de la production industrielle et l'amélioration du pouvoir d'achat en 1954 n'atténuent pas non plus les inquiétudes face aux échéances internationales. Le CNPF doute en permanence de la solidité des performances économiques françaises. En mars 1955, Georges Villiers considère que la stabilisation des prix risque d'être ébranlée par les hausses de salaires ${ }^{56}$. Il insiste sur la fragilité de l'amélioration de la position française à l'UEP. Il rappelle la nécessité de maintenir les compensations accordées aux exportateurs. Le retour à la libre négociation des salaires et la signature d'accords contractuels dans certaines branches en août 1955 sont jugés encourageants mais l'impact des fortes augmentations salariales accordées en juillet 1955 puis celui des conflits sociaux confortent la méfiance ${ }^{57}$. Georges Villiers répète que les « problèmes fondamentaux de l'économie française $»$ ne seront pas résolus sans réformes profondes ${ }^{58}$.

La formation du gouvernement de Guy Mollet en janvier 1956 puis la relance du processus inflationniste sont critiquées par le CNPF. Le premier éditorial de 1956 évoque les « sérieuses menaces » du début de la législature ${ }^{59}$. Le 20 janvier 1956, le CNPF met en garde le gouvernement contre toute résurgence de pratiques « autoritaires en matière de salaires ». En février 1956, Georges Villiers rappelle l'obligation de lier l'augmentation des salaires à celle de la productivitéc0. Les exposés de l'assemblée générale de février 1956 dénoncent les difficultés des

${ }^{53}$ G. Villiers, «L'économie française affaiblie par le dirigisme », Bulletin du CNPF, $\mathrm{n}^{\circ} 127$, décembre-janvier 1955.

${ }^{54}$ G. Villiers, «L'assemblée générale du 18 janvier 1955 », Bulletin du CNPF, n 128 , février 1955.

55 Compte rendu de l'assemblée générale, Bulletin du $C N P F, \mathrm{n}^{\circ} 129$, février 1955.

56 G. Villiers, «Les impératifs de la libéralisation des échanges », Bulletin du CNPF, $\mathrm{n}^{\circ} 130$, mars 1955 ; « Les perspectives de l'économie française et le rendez-vous d'avril », Id., n 131, avril 1955 (texte communiqué à la presse le 29 mars).

${ }^{57}$ G. Villiers, Sans titre, Bulletin du CNPF, $\mathrm{n}^{\circ} 138$, octobre 1955 ; « La stabilisation des prix et les investissements productifs, des conditions du progrès économique et social », Id., $\mathrm{n}^{\circ} 139$, novembre 1955 ; « Nécessité d'investir », Id., n 140 , décembre 1955.

58 Compte rendu de l'assemblée générale du $1^{\text {er }}$ juillet, Bulletin du CNPF, n 135 , août 1955.

${ }^{59}$ G. Villiers, «Sérieuses menaces au début de cette année et de la législature », Bulletin du $C N P F, \mathrm{n}^{\circ} 142$, janvier 1956.

${ }^{60}$ Activités du CNPF, Intervention de G. Villiers, supplément au Bulletin du CNPF, $\mathrm{n}^{\circ} 143$, février 1956. 
exportateurs français qui assistent à la fermeture des marché d'Outre-Mer sans pouvoir conquérir ailleurs de nouveaux débouchés pour leurs produits manufacturés $^{61}$. Le président de la commission des accords commerciaux Georges Morin déplore que la France soit incapable de se libérer des barrières douanières au même rythme que ses partenaires européens. Il indique que le délai qu'elle a obtenu pour abroger les principales aides aux exportations n'élimine pas les craintes face à l'accélération du processus de baisse des barrières douanières de l'OECE. Le retour des déficits français à l'UEP renforce l'inquiétude des industriels français. Le CNPF accentue ses pressions sur le gouvernement pour obtenir une augmentation de l'aide aux exportateurs et aux importateurs. Dans la première quinzaine du mois d'avril, le comité directeur puis le président de la commission de libéralisation des échanges, Roger Nathan, et une délégation patronale alertent respectivement le ministre des Finances et le président du Conseil ${ }^{62}$. Ces démarches sont vaines. La situation économique et les soldes commerciaux et financiers se dégradent. La tonalité des exposés de l'assemblée générale de juillet 1956 est alarmiste ${ }^{63}$. Georges Villiers critique « la forte augmentation des charges » liée aux mesures sociales du gouvernement. Il condamne le « dirigisme sclérosant $\gg$ des pouvoirs publics et réclame vainement une profonde réforme des institutions ${ }^{64}$. L'éditorial de septembre 1956 dénonce le blocage absolu des prix instauré le 19 juillet $1956^{65}$. En octobre, Georges Villiers invite les chefs d'entreprises à «protester énergiquement » contre l'alourdissement des charges et des dépenses publiques qui entraînent la reprise de l'inflation ${ }^{66}$. La présentation du budget de 1957 accentue la grogne ${ }^{67}$. Le 14 novembre 1956, Georges Villiers attire l'attention du ministre des Finances sur la détérioration de la balance commerciale ${ }^{68}$. Il demande de restaurer le taux de compensation des charges des exportateurs antérieur aux réductions qui l'ont amputé «alors que les salaires et la fiscalité français se sont alourdis davantage qu'à l'étranger».

L'année 1957 s'ouvre sur de sombres perspectives ${ }^{69}$. Georges Villiers considère que la situation est encore plus dégradée que ne le prévoyaient les plus pessimistes ${ }^{70}$. Pour lui, la reprise de l'inflation comme le déficit commercial empêchent la France de participer à « l'initiative hardie du Marché Commun » sans

${ }^{61}$ Numéro spécial assemblée générale, Bulletin du $C N P F, \mathrm{n}^{\circ} 145$, mars 1956.

62 Activités du CNPF, supplément au Bulletin du CNPF, $\mathrm{n}^{\circ} 149$, juillet 1956.

${ }^{63}$ Numéro spécial assemblée générale du 10 juillet, Bulletin du CNPF, n 150 , août 1956.

${ }^{64}$ G. Villiers remplace P. Ricard comme président de la commission des relations économiques internationales.

${ }^{65}$ G. Villiers, « La politique des prix du gouvernement met en péril la production », Bulletin $d u$ $C N P F, \mathrm{n}^{\circ} 151$, septembre 1956 (texte communiqué à la presse).

${ }^{66}$ G. Villiers, «La lutte contre l'inflation est incompatible avec la surcharge des prix de revient », Bulletin du CNPF $\mathrm{n}^{\circ} 152$, octobre 1956.

67 Activités du CNPF, supplément au Bulletin du CNPF, $\mathrm{n}^{\circ}$ 153, novembre 1956.

68 Activités du CNPF, supplément au Bulletin du CNPF, $\mathrm{n}^{\circ}$ 154, décembre 1956.

${ }^{69}$ G. Villiers, « Pour que la vitalité des entreprises françaises ne se heurte pas en 1957 à des difficultés encore croissantes », $\mathrm{n}^{\circ} 155$, janvier 1957.

${ }^{70}$ L'assemblée générale du CNPF et le Marché commun européen, Bulletin du CNPF, $\mathrm{n}^{\circ} 156$, février 1957. 
risquer la 《ruine $»^{71}$. Le gouvernement limite les importations pour réagir à la dégradation des soldes extérieurs le 16 mars 1957. Cette décision est critiquée par une délégation patronale reçue au ministère des Affaires Economiques ${ }^{72}$. Le 29 mars, Georges Villiers demande au président du Conseil d'augmenter sans délais l'aide à l'exportation. Le traité de Rome vient d'être signé (25 mars 1957). Il n'est pas commenté dans le Bulletin du CNPF. Les articles se concentrent sur la dégradation de la situation économique générale. Le 16 avril 1957, le comité directeur du CNPF dénonce l'absence de progrès en matière d'aide à l'exportation ou de limitation de l'inflation ${ }^{73}$. La suspension totale de la libéralisation des échanges avec l'OECE et avec la zone dollar décidée le 17 juin 1957 ne suffit pas à redresser la situation. Devant l'assemblée générale de juillet, Georges Villiers insiste sur la gravité des problèmes de trésorerie de l'Etat qui doit faire «appel à la Banque de France et prélever sur ses réserves d'or pour honorer ses échéances ${ }^{74}$. Pour lui, le dérapage des dépenses publiques depuis 1955, le sacrifice de l'investissement et l'inefficacité du «blocage des prix » expliquent la situation ${ }^{75}$. Les nouvelles restrictions imposées aux importations le 10 août 1957 n'atténuent pas l'inquiétude du CNPF. Le bureau du CNPF se réunit à titre exceptionnel le 19 août pour tenter de trouver des solutions. Il réclame une politique rigoureuse des prix dans une motion communiquée à Félix Gaillard, ministre des Affaires économiques et du plan. Le 22 août, Georges Villiers s'entretient avec Emile Hugues, secrétaire d'Etat aux Affaires économiques. Le 26, il rencontre le secrétaire d'Etat à l'industrie et au commerce. Ces démarches n'ont pas de résultats visibles ${ }^{76}$. Début septembre 1957, le bureau du CNPF dénonce le blocage des prix décidé en août. Le mois suivant, il condamne une fois de plus « le gonflement des dépenses publiques ${ }^{77}$. La mission du FMI à Paris et la décision de placer le franc sous tutelle ne sont pas commentées dans le périodique patronal. En décembre 1957, le comité directeur critique les nouveaux impôts sur la production qui sont « incompatibles avec la mise en place du Marché commun le $1^{\text {er }}$ janvier 1958 ».

Au début de l'année 1958, Georges Villiers dénonce la reprise de l'inflation ${ }^{78}$. Il critique l'instabilité gouvernementale et juge les pouvoirs publics incapables de faciliter l'engagement français en Europe ${ }^{79}$. La « gravité des perspectives économiques » conduit le comité directeur du CNPF à convoquer une assemblée générale extraordinaire le 13 mai $1958^{80}$. Les conclusions alarmistes de Georges

71 Numéro spécial assemblée générale du 15 janvier 1957, Bulletin du CNPF, n 157, mars 1957.

72 Activité du CNPF, Questions économiques internationales : Réunion du 25 mars, supplément au Bulletin du CNPF, $\mathrm{n}^{\circ}$ 158, mars 1957.

73 Travaux du comité directeur, Bulletin du CNPF, $\mathrm{n}^{\circ}$ 160, mai 1957.

${ }^{74} \mathrm{G}$. Villiers, Intervention à l'assemblée générale du $1^{\mathrm{er}}$ juillet, Numéro spécial du Bulletin du $C N P F, \mathrm{n}^{\circ} 163$, août 1957.

75 Ibid.

76 Activité du CNPF, supplément au Bulletin du CNPF, n 164, septembre 1957.

77 Activités du CNPF, « Un avertissement du CNPF », supplément au Bulletin du CNPF, $\mathrm{n}^{\circ} 166$, novembre 1957 (texte transmis à la presse).

${ }^{78}$ G. Villiers, « $1958 »$, Bulletin du CNPF, $\mathrm{n}^{\circ} 168$, janvier 1958.

79 Numéro spécial assemblée générale du 14 janvier, Bulletin du CNPF, $\mathrm{n}^{\circ} 169$, janvier 1958.

${ }^{80}$ Activités du CNPF, supplément du Bulletin du CNPF, n 173, mai 1958. 
Villiers sont transmises à la presse. Il décrit une économie menacée par l'épuisement des crédits étrangers qui financent les importations des matières premières essentielles. Il accuse les interventions arbitraires des pouvoirs publics d'avoir provoqué la situation en bloquant les « régulateurs » et en aggravant les échéances sans parvenir à défendre la monnaie ${ }^{81}$. Il réclame le rétablissement de l'équilibre budgétaire et la restauration de la liberté des marchés.

L'appel à Charles de Gaulle à la Présidence du Conseil est bien accueilli au CNPF. Lors d'une intervention télévisée, le 22 juin 1958, Georges Villiers invite à soutenir le « redressement économique » entrepris et à souscrire à l'emprunt. La politique du gouvernement est qualifiée de «constructive » lors de l'assemblée générale de juillet 1958. Le président du CNPF réclame un véritable « redressement national » pour approfondir les réformes. Bien qu'il condamne le nouvel impôt sur les sociétés adopté en août et la fragilité du rétablissement des finances extérieures, le patronat est séduit par les perspectives nouvelles de stabilité gouvernementale $^{82}$. La confiance s'affirme. Pour la première fois dans un éditorial, Georges Villiers reconnaît l'ampleur des succès des entreprises françaises en novembre $1958^{83}$. La réforme monétaire est considérée comme un signe complémentaire de la volonté politique de rétablir les équilibres ${ }^{84}$.

\section{LE CNPF, UN SOUTIEN SANS FAILLE À LA RÉPUBLIQUE GAULLIENNE (1959-1969) ?}

En janvier 1959, Georges Villiers accorde sa « confiance franche » aux nouveaux dirigeants ${ }^{85}$. Le report de l'assemblée générale du CNPF de quelques semaines puis l'absence de critique contre les pouvoirs publics révèlent que le patronat attend avec espoir l'application des projets gouvernementaux ${ }^{86}$. En mai, Georges Villiers demande à chacun de soutenir les réformes afin de consolider l'amélioration de la situation financière et monétaire ${ }^{87}$. Il rompt la trêve en octobre en dénonçant la reprise de l'inflation et les risques de gonflement des dépenses publiques qui fragilisent la balance commerciale ${ }^{88}$. Pour éviter le double écueil de la récession et celui de l'inflation, Georges Villiers réclame d'appliquer une politique résolument plus libérale et de lutter avec vigueur contre l'alourdissement des prix de revient. Le Premier ministre Michel Debré répond à Georges

${ }^{81}$ Numéro spécial assemblée générale extraordinaire, Bulletin du $C N P F, \mathrm{n}^{\circ} 174$, juin 1958.

82 Activité du CNPF, supplément au Bulletin du CNPF, n 176, août-septembre 1958 ; G. Villiers, «Définir une politique économique à long terme », Id., n 177 , octobre 1958.

${ }^{83}$ G. Villiers, «Les chances de l'économie française », Bulletin du CNPF, $\mathrm{n}^{\circ} 178$, novembre 1958.

${ }^{84}$ G. Villiers, « Exigences d'une économie moderne », Bulletin du CNPF, $\mathrm{n}^{\circ} 179$, décembre 1958.

${ }^{85}$ G. Villiers, « $1959 »$, Bulletin du CNPF, $\mathrm{n}^{\circ} 180$, janvier 1959.

${ }^{86}$ G. Villiers, « Notre prochaine assemblée générale », Bulletin du CNPF, n 181, février 1959.

${ }^{87}$ G. Villiers, « Exporter, investir pour préparer de nouveaux progrès économiques et sociaux », Bulletin du CNPF, $\mathrm{n}^{\circ} 185$, mai 1959.

${ }^{88}$ G. Villiers, « Pour que s'affirme l'expansion », Bulletin du CNPF, $\mathrm{n}^{\circ} 189$, octobre 1959. 
Villiers le 12 novembre $1959^{89}$. Il s'engage à garantir la stabilité des prix et à développer l'épargne et la productivité « sans taxation ni subvention ». Ces objectifs correspondent aux demandes du CNPF.

La création du Nouveau franc le $1^{\text {er }}$ janvier 1960 confirme la volonté de réformes. La forte croissance des exportations repousse le spectre de la récession. Le patronat est enclin à l'optimisme. Son bulletin s'affiche désormais comme le porte-parole du Patronat français. Ce changement de titre ne modifie pas radicalement la stratégie patronale. Même s'il invite le CNPF à abandonner ses « positions purement revendicatives » et à approfondir sa doctrine au début de 1960, Georges Villiers ne change pas de discours ${ }^{90}$. Il réclame toujours aux gouvernants d'éliminer les menaces inflationnistes en limitant les hausses salariales et en réformant en profondeur les services publics ${ }^{91}$. Il demande aux entreprises de prolonger leurs efforts pour améliorer la situation économique ${ }^{92}$. Le CNPF reste sur la défensive lorsqu'il dénonce la décision des six pays du Marché commun d'accélérer le processus de désarmement douanier en mai $1960^{93}$. Georges Villiers considère que cette décision fait courir des « risques inutiles » aux entreprises françaises alors qu'aucun progrès notable en matière d'harmonisation des conditions de la concurrence entre les Six n'a été réalisé. Plusieurs études du CNPF affirment que l'alourdissement des charges des producteurs français depuis 1952 constitue un obstacle essentiel à l'expansion économique ${ }^{94}$. Ces études ne tiennent pas compte de la capacité des entreprises françaises à réagir à l'ouverture des frontières que révèlent le redressement des soldes extérieurs et la reprise de la croissance. Pour la première fois depuis 1927, la balance commerciale française est équilibrée en 1960. Le CNPF continue pourtant à insister sur la fragilité des améliorations de la conjoncture ${ }^{95}$. Le seul changement positif qui est présenté sans nuance à la fin de 1961 concerne la participation du patronat aux travaux du nouveau Conseil supérieur du Plan. Georges Villiers affirme qu'il s'agit là d'un élément essentiel de la modernisation économique ${ }^{96}$.

${ }^{89}$ M. Debré, «Les conditions de la stabilité des prix », Bulletin du CNPF, $\mathrm{n}^{\circ}$ 191, décembre 1959.

${ }^{90}$ G. Villiers, « Notre prochaine assemblée générale le 16 février », Patronat français $\mathrm{n}^{\circ} 193$, février 1960.

91 G. Villiers, « 1960 », Patronat français, n 192, janvier 1960 ; intervention à l'assemblée générale de janvier 1960, numéro spécial, n 194, mars 1960 ; «Graves problèmes : Algérie, Europe, paix du monde », Id., n² 203, janvier 1961.

92 G. Villiers, "Conclusions de l'assemblée générale du 17 janvier », Patronat français, $\mathrm{n}^{\circ}$ 204, février 1961 (texte transmis à la presse) ; Numéro spécial assemblée générale, Id., $\mathrm{n}^{\circ} 209$, juillet 1961.

93 G. Villiers, «Conclusion de l'assemblée générale », Patronat français, n 198, juillet 1960.

94 Non signé, «Le budget de l’Etat entre 1952 et 1960 », supplément de Patronat français, n 198 , juillet 1960 ; Compte rendu des travaux de la commission de politique économique générale du CNPF réunie le 17 octobre 1960, «Le rapport Rueff Armand », Id., Numéro spécial, octobre 1960.

95 G. Villiers, « La concurrence », Patronat français, n² 200, octobre 1960 ; « Le marché commun, impératif essentiel de la politique économique française », Id., n² 201, novembre 1960.

96 G. Villiers, « Notre pays doit prendre conscience des possibilités et des conditions de progrès d'une économie moderne », Patronat français, n² 212, novembre 1961. 
Le doute sur la solidité de la croissance économique et de l'excédent commercial se confirme au cours de l'année 1962. Le patronat dénonce les objectifs excessifs du quatrième Plan ${ }^{97}$. Les dispositions adoptées pour stimuler l'investissement sont jugées très insuffisantes. Les inquiétudes sont d'autant plus vives que la diminution des droits internes au Marché commun est fixée à juillet 1963 et que les négociations du GATT laissent présager d'une forte diminution des barrières douanières à brève échéance ${ }^{98}$. Georges Villiers demande vainement aux négociateurs français de ménager des transitions pour faciliter l'adaptation des entreprises. En janvier 1963, il lance un cri d'alarme en constatant la reprise de l'inflation et la dégradation de la balance commerciale 《 après deux ans de rapides progrès ${ }^{99}$. Il réclame aux pouvoirs publics de lutter contre l'inflation et de stimuler l'investissement afin de permettre à l'industrie de développer ses performances techniques et d'exporter ${ }^{100}$. En juillet, Georges Villiers répète cet appel en insistant sur la nécessité d'écarter toute «action dirigiste sur les prix » et toute "fiscalité anti-économique ${ }^{101}$. La reprise de l'inflation et l'augmentation des importations industrielles françaises se confirment durant l'été. Georges Villiers accuse les pouvoirs publics d'avoir contribué à élargir les différences de prix de revient entre la France et ses partenaires comme avant les réformes de $1958^{102}$. Le patronat est en partie satisfait par le plan de stabilisation adopté en septembre $1963^{103}$. Il critique cependant l'insuffisance des mesures adoptées pour limiter les hausses de salaires et les dépenses budgétaires. Le 21 novembre 1963, le CNPF dénonce le blocage des prix qui «empêche les adaptations nécessaires au commerce extérieur » et affaiblit les secteurs dont les prix restent libres ${ }^{104}$. Le redressement de la conjoncture économique et la « limitation de l'inflation budgétaire » redonnent confiance au patronat. Devant l'assemblée générale de janvier 1964, Georges Villiers invite à prolonger les efforts pour consolider la monnaie et répondre aux exigences de « l'ouverture des frontières ${ }^{105}$. Il encourage une nouvelle fois les organisations professionnelles à participer activement à la résolution des problèmes ${ }^{106}$.

${ }^{97}$ G. Villiers, «L'année s'ouvre sur les perspectives du quatrième plan », Patronat français, $\mathrm{n}^{\circ} 214$, janvier 1962 .

${ }^{98}$ G. Villiers, « Perspectives mondiales », Patronat français, ${ }^{\circ}$ 224, décembre 1962.

${ }^{99}$ G. Villiers, Déclaration devant l'assemblée générale de janvier, Patronat français, $\mathrm{n}^{\circ} 226$, février 1963 (texte transmis à la presse).

${ }^{100}$ G. Villiers, «Conclusions générales », Numéro spécial assemblée générale du 15 janvier, Patronat français, $\mathrm{n}^{\circ} 227$, mars 1963.

${ }^{101}$ G. Villiers, « Conclusions », Numéro spécial assemblée générale, Patronat français, $\mathrm{n}^{\circ} 231$, juillet 1963.

${ }^{102}$ G. Villiers, « Le commerce extérieur tend à se détériorer », Patronat français, $\mathrm{n}^{\circ} 232$, août-septembre 1963.

${ }^{103}$ G. Villiers, « La commission des prix examine les conséquences du blocage des prix industriels à la production du 12 septembre », Patronat français, $n^{\circ} 233$, octobre 1963.

${ }^{104}$ Communiqué de presse à l'issue de la réunion du comité directeur du 21 novembre 1963, Patronat français, $\mathrm{n}^{\circ} 235$, décembre 1963.

${ }^{105}$ G. Villiers, « Conclusions », Numéro spécial assemblée générale du 14 janvier, Patronat français, n²37, décembre 1963.

${ }^{106}$ G. Villiers, «L'issue de la lutte contre l'inflation dépend aussi de réalisations constructives », Patronat français, n $^{\circ} 239$, avril 1964 ; Numéro spécial assemblée générale du 16 juin consacré à la 
Un an plus tard, le CNPF présente un véritable programme économique ${ }^{107}$. Il préconise de stimuler l'investissement productif et l'exportation en allégeant la fiscalité, en libéralisant les prix et en cantonnant l'Etat à un rôle d'orientation et d'encadrement de l'activité. Ce programme encourage le patronat à critiquer la politique gouvernementale. En avril 1965, Georges Villiers explique le ralentissement de la croissance et la dégradation des soldes extérieurs par l'augmentation des prix de revient industriels et par la faiblesse de l'investissement ${ }^{108}$. Il considère que ni la baisse du taux d'escompte, ni la promesse d'une réforme fiscale ne suffiront à relancer l'investissement ${ }^{109}$. Le CNPF reconnaît le bien-fondé de l'augmentation du prix de l'électricité décidée en juillet mais il met en garde contre les difficultés qu'elle risque de produire dans le secteur privé dont les prix restent bloqués. Même si les mesures de relance de l'activité présentées par le Premier ministre le 15 septembre 1965 sont bien accueillies au CNPF, elles n'éliminent ses critiques. Le maintien du blocage des prix reste le motif de discorde essentiel. En septembre 1965, un rapport de la commission des prix le condamne et Georges Villiers le dénonce dans une lettre au Premier ministre ${ }^{110}$. En novembre, Georges Villiers insiste sur l'obligation de renoncer au blocage des prix avant la libéralisation des changes et des mouvements de capitaux prévue en janvier $1967^{111}$.

La reprise de l'expansion économique et la stabilisation des prix sont jugés trop fragiles pour que le CNPF puisse souscrire à la décision des Six d'avancer au $1^{\text {er }}$ juillet 1968 la suppression totale des droits intérieurs pour les produits industriels ${ }^{112}$. L'élection de Pierre Huvelin à la présidence du CNPF le 14 juin 1966 ne modifie pas le discours patronal ${ }^{113}$. Son premier éditorial souligne la fragilité de la conjoncture française et réclame de libérer les prix et de réduire les charges ${ }^{114}$. Il insiste comme son prédécesseur sur la nécessité de répercuter dans les prix de vente des entreprises l'inévitable « réajustement des tarifs publics ». Pierre Huvelin définit l'échéance de juillet 1968 comme une priorité absolue ${ }^{115}$.

situation de la France dans le Marché commun., Id., n 242, juillet 1964 ; G. Villiers, « Face à la concurrence américaine les entreprises européennes devront concentrer leurs moyens », Id., $\mathrm{n}^{\circ} 243$, août-septembre 1964 ; "Nécessité de la Communauté économique européenne », Id., n 246, décembre 1964.

${ }^{107}$ Numéro spécial assemblée générale du 19 janvier, Patronat français, $n^{\circ}$ 248, février 1965.

${ }^{108}$ G. Villiers, Patronat français, ${ }^{\circ} 250$, avril 1965.

${ }^{109}$ G. Villiers, «Conclusions de l'assemblée générale », Patronat français, n 253, juillet 1965.

${ }^{110}$ « Rapport sur l'abolition du dirigisme des prix », Patronat français, n 260, mars 1966.

${ }^{111}$ G. Villiers, « La fin de la période transitoire du Marché commun approche, serons-nous prêts en 1970 ? », supplément de Patronat français, n 256, novembre 1965.

${ }^{112}$ G. Villiers, «Conclusions présentées à l'assemblée générale le 18 janvier 1966 », supplément de Patronat français, ${ }^{\circ}$ 259, février 1966 ; Bureau du CNPF, communiqué de presse du 11 mai 1966, Id., $\mathrm{n}^{\circ} 263$, juin 1966 .

${ }^{113}$ Numéro spécial assemblée générale, Patronat français, n 264, juillet 1966.

${ }^{114}$ P. Huvelin, « L'insuffisance des marges bénéficiaires demeure le principal obstacle aux perspectives de progrès de l'économie française », Patronat français, $\mathrm{n}^{\circ}$ 265, août-septembre 1966.

${ }^{115}$ P. Huvelin, «Les entreprises françaises engagées dans une très dure compétition veulent gagner la partie », supplément de Patronat français, n $^{\circ}$ 267, novembre 1966. 
En février 1967, il réclame au gouvernement de relancer l'investissement et de restaurer les capacités financières des entreprises pour rattraper les retards provoqués par le plan de stabilisation de $1963^{116}$. La critique de l'alourdissement du « fardeau des charges » est d'autant plus vive en juillet 1967 que les performances françaises à l'étranger se dégradent ${ }^{117}$. Pierre Huvelin réclame au gouvernement de garantir les équilibres généraux afin de permettre à la France de surmonter les proches échéances de baisse des droits internes à l'Europe et aux signataires du GATT. Il précise que l'Etat sera d'autant plus efficace « qu'il évitera de multiplier ses interventions ». Ce discours a peu d'effets. Le 17 octobre 1967, le comité directeur du CNPF condamne l'augmentation des cotisations sociales et des tarifs publics $^{118}$. Il dénonce l'insuffisance des ordonnances gouvernementales susceptibles d'aider les entreprises à surmonter la suppression des droits entre les Six. La dévaluation de la livre sterling et l'introduction de la TVA en Allemagne renforcent les critiques à la fin de 1967.

Le plan de relance Debré présenté en janvier 1968 répond aux demandes immédiates du CNPF mais les dispositions concernant les investissements et la fiscalité déçoivent rapidement ${ }^{119}$. Dans un entretien pour l'hebdomadaire La Vie française, Pierre Huvelin demande au gouvernement de « rendre aux entreprises françaises la capacité de maîtriser leurs prix ${ }^{120}$. Il explique les difficultés chroniques du commerce extérieur français par la « lourdeur et l'anachronisme de l'interventionnisme ». Il réclame l'instauration d'un «climat favorable à l'initiative et à l'esprit d'entreprise pour surmonter la crise monétaire et la concurrence étrangère ». Patronat français maintient ses pressions sur les pouvoirs publics pour préparer l'échéance de l'ouverture des frontières du $1^{\text {er }}$ juillet ${ }^{121}$. Les effets des mouvements de mai-juin retiennent ensuite toute l'attention. Le comité directeur du CNPF met en garde contre les conséquences des hausses de salaires sur le chômage et sur l'inflation ${ }^{122}$. Il condamne le rétablissement du contrôle des changes qu'il juge incapable de « ranimer la confiance». En juillet 1968, Pierre Huvelin attribue la crise du printemps à « la prolifération d'une bureaucratie paperassière et centralisatrice étouffant l'esprit d'initiative ${ }^{123}$. Il réclame une réduction immédiate et massive des charges annexées aux salaires et des « efforts audacieux » pour limiter les hausses de prix. Le gouvernement de Maurice Couve de Murville formé le 10 juillet est soutenu par le CNPF lorsqu'il annonce la suppression du

\footnotetext{
${ }^{116}$ Numéro spécial assemblée générale, Patronat français, $\mathrm{n}^{\circ}$ 270, février 1967.

${ }^{117}$ P. Huvelin, Patronat français, ${ }^{\circ} 271$, avril 1967 ; «Conclusions de l'assemblée générale », Id., $\mathrm{n}^{\circ} 275$, juillet 1967 .

${ }^{118}$ Travaux du bureau du CNPF, « Motion », supplément de Patronat français, ${ }^{\circ} 278$, novembre 1967.

${ }^{119}$ P. Huvelin, « Le plan de relance n'est pas à la mesure du problème des investissements productifs », supplément de Patronat français, $\mathrm{n}^{\circ} 282$, mars 1968.

${ }^{120}$ Interview de P. Huvelin, Patronat français, $\mathrm{n}^{\circ} 283$, avril 1968.

${ }^{121}$ Non signé, « La double échéance du désarmement douanier industriel entre les Six et de l'application des accords Kennedy », Patronat français, n² 284, mai 1968.

${ }^{122}$ Travaux du bureau du CNPF, « Les conséquences des mouvements de mai-juin 1968 », supplément de Patronat français, $\mathrm{n}^{\circ} 285$, juin-juillet 1968 .

${ }^{123}$ Numéro spécial assemblée générale, Patronat français, nº 286, juillet 1968.
} 
contrôle des changes ${ }^{124}$. Il est condamné quelques jours plus tard parce qu'il ne parvient pas à enrayer l'augmentation des charges salariales ${ }^{125}$. Le 25 novembre, le CNPF insiste sur la nécessité de défendre le franc en développant les investissements productifs. Pierre Huvelin et le bureau du CNPF critiquent ensuite la hausse du taux d'escompte qui fait monter les prix de revient, casse l'expansion et fragilise la monnaie ${ }^{126}$. Un autre communiqué de presse le 21 décembre dénonce le « relèvement de la TVA » qui annule les effets de la suppression de la taxe sur les salaires et qui est incapable d'enrayer « la crise de confiance ». La croissance des prélèvements obligatoires et des salaires, le « déficit de la balance commerciale » et la fragilité du franc constituent les principales préoccupations patronales du début de l'année $1969^{127}$. Le maintien du contrôle des changes est jugé «incompatible avec l'exercice normal du commerce extérieur ${ }^{128}$. Dans un article publié dans Le Monde en mai 1969, Ambroise Roux, président de la commission économie générale du CNPF, condamne les charges imposées aux industriels ${ }^{129}$. L'éditorial de Patronat français de juin puis les exposés de l'assemblée générale de juillet affirment leur satisfaction face au record absolu de la production industrielle tout en insistant avec force sur la persistance des menaces inflationnistes ${ }^{130}$. Pierre Huvelin fait une conférence de presse à l'annonce de la dévaluation du franc du 11 août 1969. Il indique que la décision «ne saurait apporter solution au problème de fond » mais qu'elle doit être assortie d'une «gestion rigoureuse des budgets, de l'allégement des contraintes administratives et de l'élimination des dépenses stériles » afin de « rétablir un équilibre véritable ${ }^{131}$. L'engagement du gouvernement en faveur d'une réduction du blocage des prix et d'un plan d'assainissement sont très bien accueillis. Les pressions patronales vont aboutir. Comme le confirme la réforme du CNPF présentée à l'assemblée générale extraordinaire convoquée le 28 octobre 1969, le CNPF n'est plus seulement un « comité de liaison», il est devenu « un promoteur de l'entreprise quel que soit son secteur ». En se posant en défenseur de l'entreprise et du franc il s'est affirmé comme un groupe de pression influent.

\section{CONCLUSION}

Les redondances du discours du CNPF entre 1947 et 1969 s'expliquent par une prise de position marquée en faveur du libéralisme. L'affirmation d'une nou-

\footnotetext{
${ }^{124}$ Travaux du bureau du CNPF, supplément de Patronat français, $\mathrm{n}^{\circ} 288$, octobre 1968 (texte communiqué à la presse le 6 septembre).

${ }^{125} \mathrm{P}$. Huvelin, supplément de Patronat français, $\mathrm{n}^{\circ} 287$, août-septembre 1968.

${ }^{126}$ P. Huvelin, Patronat français, $n^{\circ} 290$, décembre 1968 (transmis à la presse).

${ }^{127}$ Numéro spécial assemblée générale du 14 janvier, supplément de Patronat français, $\mathrm{n}^{\circ} 292$, février 1969.

${ }^{128}$ P. Huvelin, Patronat français, $\mathrm{n}^{\circ} 294$, avril 1969.

${ }^{129}$ A. Roux, Patronat français, $\mathrm{n}^{\circ} 295$, mai 1969.

${ }^{130}$ P. Huvelin, supplément de Patronat français, $\mathrm{n}^{\circ}$ 296, juin 1969 ; Conclusions de l'assemblée générale, Id., n² 297, juillet 1969.

${ }^{131}$ P. Huvelin, supplément de Patronat français, n 298, août-septembre 1969.
} 
velle génération de chefs d'entreprises plus au fait des réalités de l'économie moderne élargit le registre thématique sans modifier l'orientation générale du discours. La critique des charges et des déficits de toutes natures reste systématique. Elle s'accompagne d'une demande permanente de protections et de soutiens pour permettre aux entreprises de résister à l'ouverture des frontières. En réclamant ce qu'ils condamnent, les dirigeants patronaux défendent l'entreprise contre les maladresses des pouvoirs publics qui condamnent le franc.

Le discours patronal tarde à reconnaître la force de l'expansion économique et la capacité des entreprises à tirer profit de la construction européenne. Ce manque de clairvoyance s'explique par le caractère obsessionnel de ses critiques contre les pouvoirs publics et par l'ampleur et la rapidité des changements de l'économie française entre 1947 et 1969.

A la fin des années 1960, le CNPF est animé par une nouvelle génération de chefs d'entreprises favorables à la concertation et disposant de relais dans les foyers de la décision politique. Il représente le fleuron de l'industrie française défendu par le second président de la $\mathrm{V}^{\mathrm{e}}$ République Georges Pompidou. La contrainte extérieure, la défense du franc et la modernisation sont devenues les impératifs gouvernementaux comme le préconisaient les pionniers du CNPF dès que la question de l'ouverture des frontières a été posée. Les décideurs économiques et politiques ont désormais des ambitions communes. Ils entretiennent des relations étroites. La convergence de leurs aspirations et l'imbrication de leurs réseaux résultent des efforts entrepris par les fondateurs du CNPF depuis sa création.

\section{SOURCES ET BIBLIOGRAPHIE}

CNPF, Bulletin du CNPF puis Patronat français, 1947-1970. Publication mensuelle à partir d'avril 1954. Sauf indication contraire, les références aux articles de Georges Villiers puis de Pierre Huvelin sont extraites de leurs éditoriaux.

Ehrmann, Henry, La politique du patronat français. 1936-1955, Paris, Armand Colin, 1959.

Touchelay, Béatrice, « Le CNPF et l'INSEE entre 1946 et 1961 : histoire d'une alliance modernisatrice », Le Mouvement Social, $\mathrm{n}^{\circ} 191$, avril-juin 2000.

Weber, Henri, Le parti des patrons. Le CNPF : 1946-1990, Paris, Seuil, 1996. 